\title{
Osteosynthese distaler Radiusfrakturen mit der Kirschner-Draht-Osteosynthese
}

\author{
Thomas Klaus Hotz
}

\section{Zusammenfassung}

Die Kirschner-Draht-Osteosynthese stellt ein Bindeglied zwischen der konservativen Frakturbehandlung distaler Radiusfrakturen und den operativen Behandlungsmethoden wie Platte oder Fixateur externe dar. Die Methode ist minimal invasiv, einfach, billig und schnell. Die Indikation für die Kirschner-Draht-Osteosynthese besteht bei distalen Monoblock-Frakturen mit Dislokation nach dorsal und ohne wesentliche Gelenkbeteiligung.
Der Eingriff muss in einer exakten Operationstechnik erfolgen um Komplikationen zu vermeiden. Die Kirschner-Drähte werden unter Bildwandlerkontrolle durch nur kleine Hautinzisionen mit Vorteil in den Frakturspalt eingebracht und die Fraktur gegen eine Sekundärdislokation abgestützt. Nach 4-6 Wochen Ruhigstellung in Gips oder Handgelenksmanschette können die Kirschner-Drähte nach 8 Wochen in Lokalanästhesie entfernt werden.

Im mitteleuropäischen Raum hat sich vor allem die von der Arbeitsgemeinschaft für Osteosynthesefragen (AO) vorgeschlagene perkutane Methode durchgesetzt. Diese Methode wurde unter anderem 1976 durch Kapandji mittels intrafokaler Führung der Spickdrähte modifiziert. Während Kapandji seine Methode vor allem für junge Patienten beschrieb und eine funktionelle Nachbehandlung anschloss, wurde diese Methode wiederum durch verschiedene Anwender weiterentwickelt, so dass mittlerweilen vorwiegend auch alte Patienten behandelt werden unter Zuhilfenahme von drei statt zwei Kirschner-Drähten.

Die modifizierte Kapandji-Technik stellt heutzutage bei extraartikulären distalen Radiusfrakturen, bei denen ein genügend stabiler Monoblock des gelenktragenden Frakturfragmentes vorliegt, eine interessante Alternative zu den anderen Osteosynthese-Verfahren dar. Die Methode besticht durch eine wenig invasive Weichteilbehandlung, eine einfache und billige Osteosynthese-Technik sowie durch die Möglichkeit der Kirschner-Draht-Entfernung in Lokalanästhesie.

Wichtig für den erfolgreichen Einsatz dieser Methode sind eine sorgfältige Operationstechnik und eine korrekte Nachbehandlung. Die Kenntnis der Gefahren und Komplikationen spielen ebenso eine entscheidende Rolle (Abb.2-4). werden. Die zusätzliche KirschnerDraht-Osteosynthese richtet sich vor allem an diejenigen Frakturen, bei welchen eine Re-Dislokation bei alleiniger Gipsfixation droht.

Seit ihrer Beschreibung durch Lambotte 1908 hat sich die Kirschner-Draht-Osteosynthese als Mittelweg zwischen der konservativen Behandlung distaler Radiusfrakturen und den Osteosynthesetechniken durch Fixateur externe oder Platten herauskristallisiert. Die Kirschner-Draht-Osteosynthese stellt in ihren Variationen eine wenig invasive, einfache und billige Osteosynthese-Technik dar. Diese Methode der Retention einer zuvor reponierten Fraktur hat eine lange Tradition und ist in diversen Varianten in Anwendung (Abb.1).

OP-JOURNAL 2003; 19: 21-27

(c) Georg Thieme Verlag Stuttgart . New York

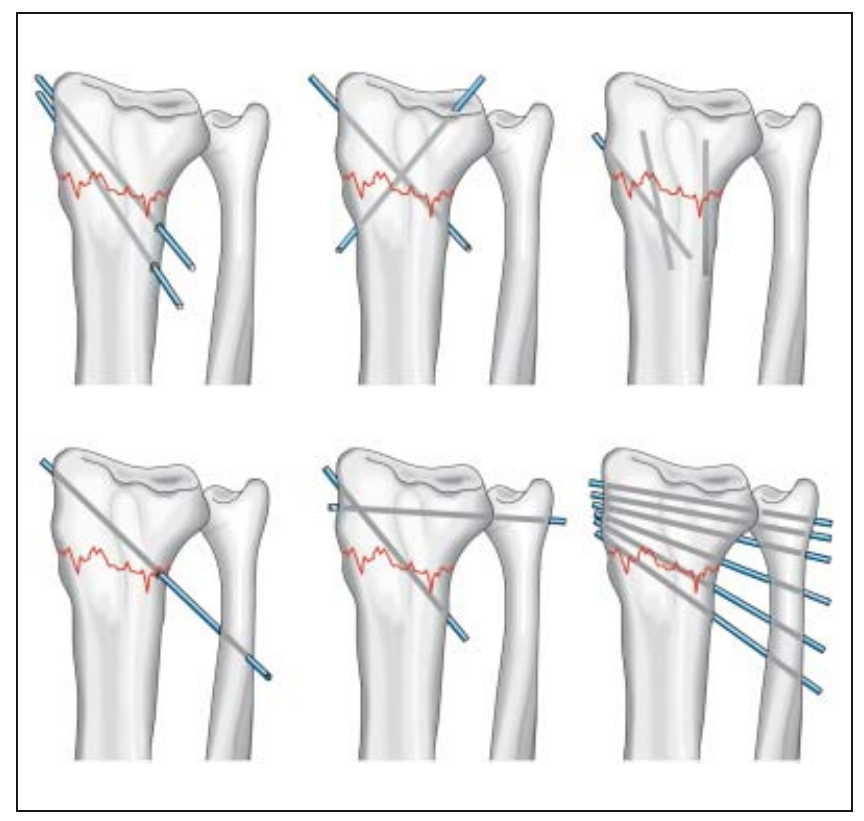

Abb. 1 Methoden der Kirschner-DrahtOsteosynthesen am distalen Radius. 

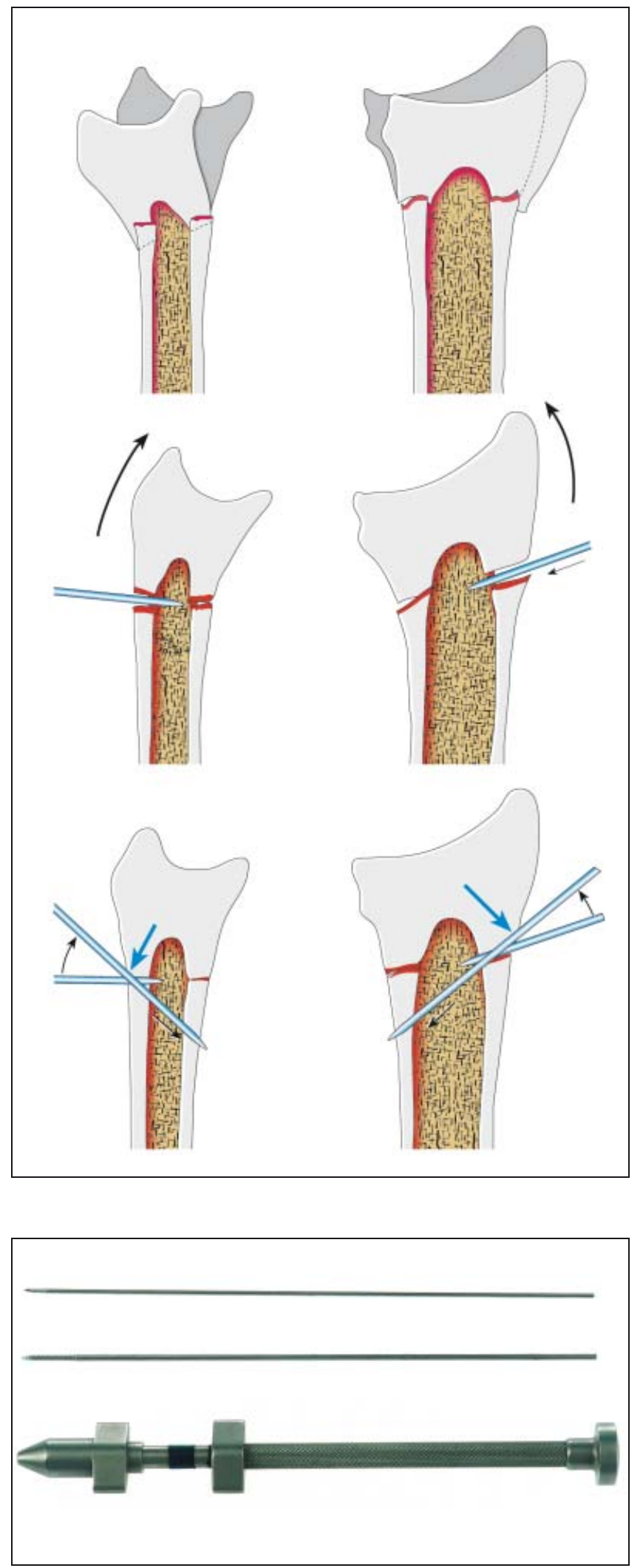

Abb. 6 1,6 mm Gewinde-Kirschner-Drähte mit kurzem Gewinde (oben) resp. langem Gewinde für besseren Halt in der Gegenkortikalis (Mitte). Handbohrfutter für schonendes Einbringen der Drähte (unten).
Abb.5b KD-Osteosynthese nach Kapandji.

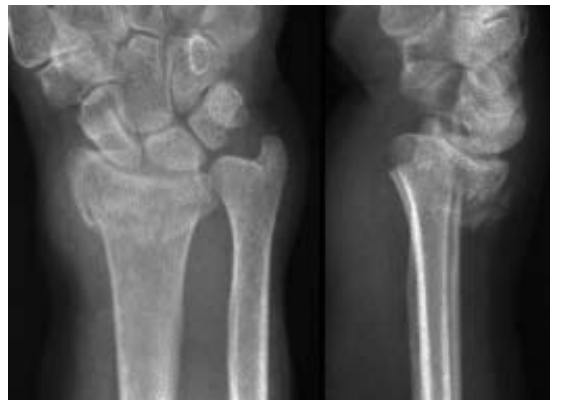

Abb. 7 66jährige Frau, Sturz auf Strasse - Unfallbild.

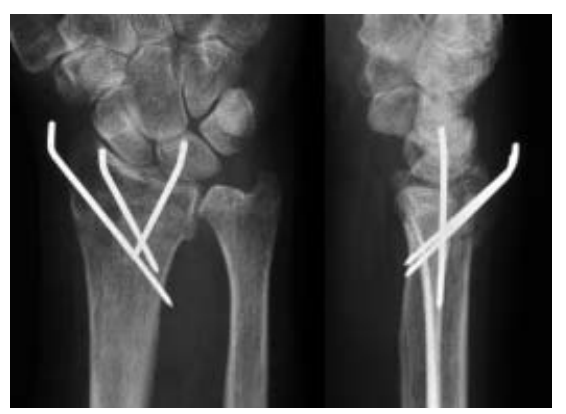

Abb. 86 Wochen nach intrafokaler KD-Osteosynthese nach Kapandji.

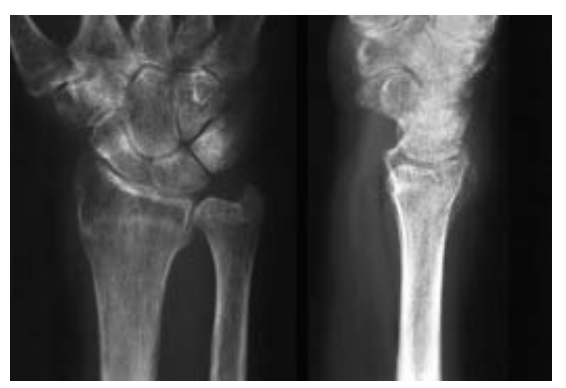

Abb. 9 Kontrollbild 5 Monate nach Unfall nach 8 Wochen KD-Entfernung.

Hauptgruppen: Junge Patienten mit nach dorsal dislozierten Frakturen und ältere Patienten mit Osteoporose und entsprechenden dorsalen Trümmerzonen.

Kontraindikationen bestehen bei Dislokationen nach palmar, intraartikulären Impressionen sowie ausgedehnten Trümmerfrakturen.

Zur Kirschner-Draht-Osteosynthese werden 1,6 mm Gewinde-Kirschner-Drähte aus Stahl verwendet. Diese sind im Handel mit kurzem Gewinde (ca. 5 mm) oder langem Gewinde (ca. $12-15 \mathrm{~mm}$ ) erhältlich. Um einen sicheren Halt in der Gegenkortikalis für die Kirschner-Drähte sicherzustellen, müssen lange Gewinde verwendet werden. Zum Vermeiden von Hitzeschäden am Knochen oder Leerdre- 
hen des Gewindes in der Gegenkortikalis, was in beiden Fällen zu einer vorzeitigen Lockerung der Kirschner-Drähte führen kann, wird mit Vorteil ein Handbohrfutter und nicht eine Bohrmaschine verwendet. Letztere sollte nur in Ausnahmefällen und dann nur mit sehr niedriger Drehzahl verwendet werden (Abb.6-9).

Einbringen der Kirschnerdrähte von Hand anstelle mit einer Bohrmaschine bewahrt vor einem Ueberdrehen des Gewindes oder Hitzeschäden am Knochen und hilft somit eine vorzeitige Auslockerung der Drähte zu vermeiden.

\section{Operationstechnik}

Nach sterilem Abdecken wird unter Bildwandlerkontrolle als erstes eine geschlossene Reposition der Fraktur in möglichst anatomisch korrekter Stellung der Hauptfragmente durchgeführt. Teilweise ist dazu ein Hypomochlion, zum Beispiel in Form einer straff gewickelten Tuchrolle notwendig. Je genauer die Reposition erfolgen kann, desto einfacher gestaltet sich das anschließende Einführen der Kirschner-Drähte (Abb.10).

Bei starker Weichteilschwellung, unsicherer Anatomie oder ungenügender Erfahrung empfiehlt sich als nächster Schritt unter Bildwandlerkontrolle und Zuhilfenahme einer röntgendichten Klemme oder Ähnlichem das Einzeichnen der Kirschner-Draht-Eintrittsstellen auf der Haut: In der a.p.-Projektion je radial und ulnar des Tuberkulum listeri sowie die dritte Inzisionsstelle in der seitlichen Projektion genau in der Mitte des Radiusschaftes.

Die Hautinzisionen erfolgen von Höhe Bruchspalt nach distal über eine Strecke von ca. $10 \mathrm{~mm}$, anschließend wird mit zwei spitzen, $90^{\circ}$ zueinander geführten Moskitoklemmen stumpf bis auf den Bruchspalt präpariert. Mit dieser Methode werden Verletzungen von Nerven und Sehnen weitestgehend vermieden. Das Erreichen des Bruchspaltes kann getastet werden, gleichzeitig kommt es regelmä- ßig zum Austritt von Frakturhämatom, vermischt mit Fettaugen (Abb.11).

Je nach Frakturverlauf wird zuerst durch die mittlere Inzision unter Aufspreizen der Weichteile durch die Moskitoklemmen ein erster Kirschner-Draht rechtwinklig zum Radiusschaft in den Frakturspalt eingeführt und anschließend durch Aufstellen des Kirschner-Drahtes unter Zug am Handbohrfutter nach distal das distale Frakturfragment aufgehebelt und anschließend der Gewinde-Kirschnerdraht in einem Winkel von etwa $45^{\circ}$ zur Radiusschaftachse durch Druck und Drehbewegungen in der Gegenkortikalis verankert bis er diese vollständig durchbohrt hat. Analog werden der zweite Draht radial und der dritte ulnar eingebracht und jeweils die Fraktur, soweit nötig, zusätzlich nochmals aufgehebelt (Abb.12, Abb.16).

Durch das intrafokale Einbringen der Kirschnerdrähte können diese als Repositionshilfe gebraucht werden
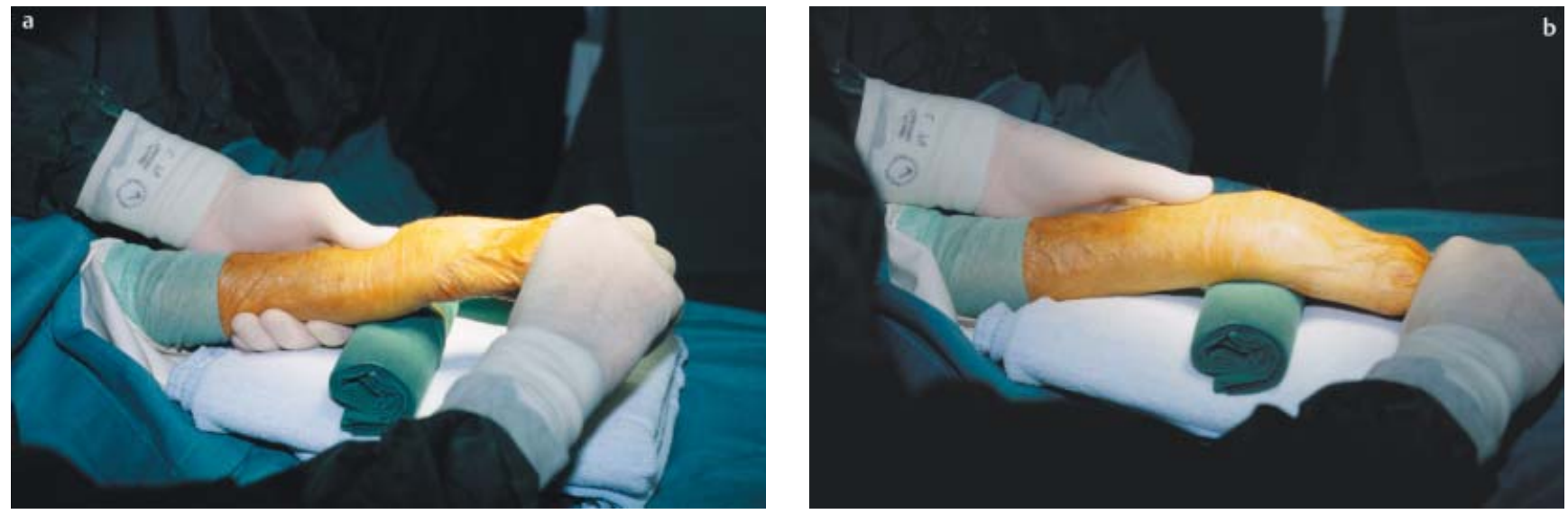

Abb.10 Geschlossene Reposition (a) und über Tuchrolle (b) Retention.

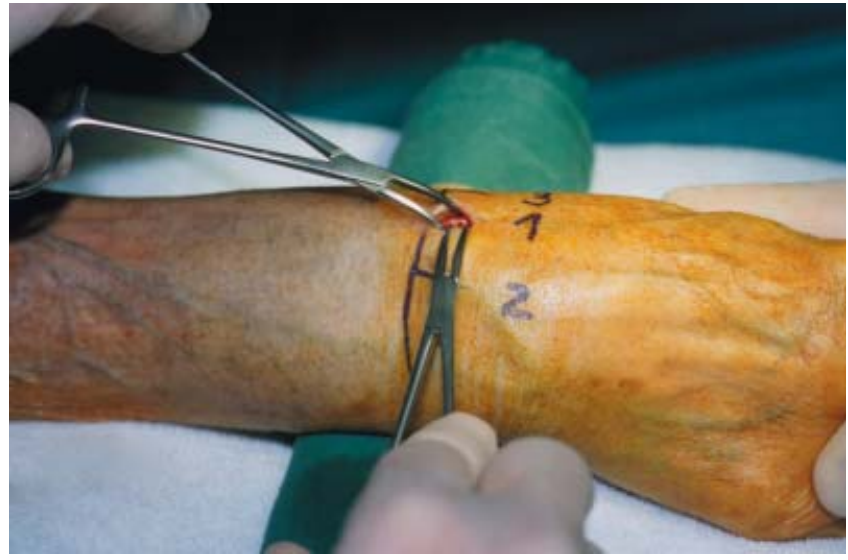

Abb.11 Stumpfe Präparation bis auf den Bruchspalt mit Klemmen.

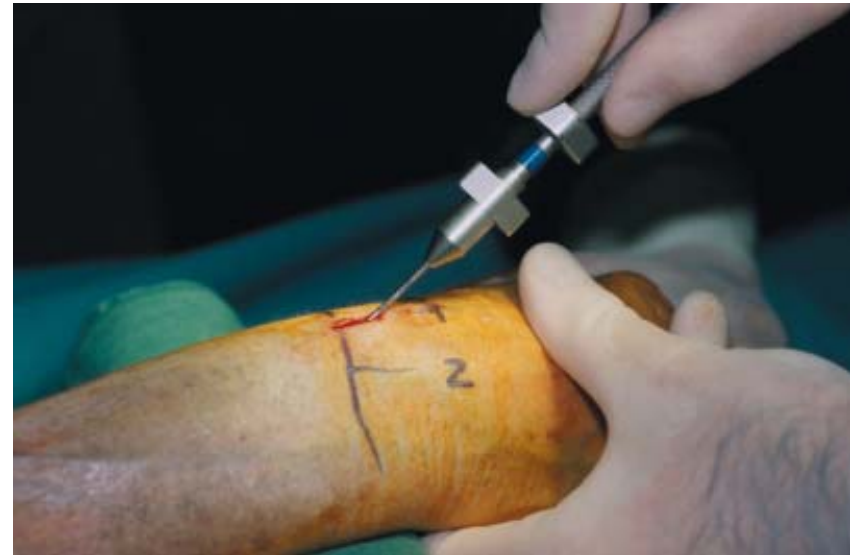

Abb.12 Nach zusätzlichem Aufhebeln der Fraktur, Eindrehen des Kirschner-Drahtes in die Gegenkortikalis. 


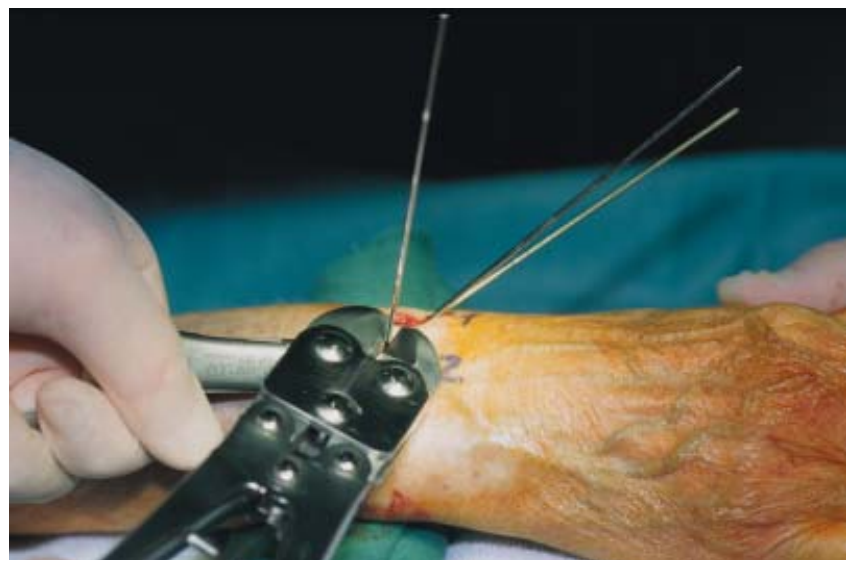

Abb.13 Kürzen des nach dorsal umgebogenen Kirschner-Drahtes und anschließend Drehen um $180^{\circ}$.

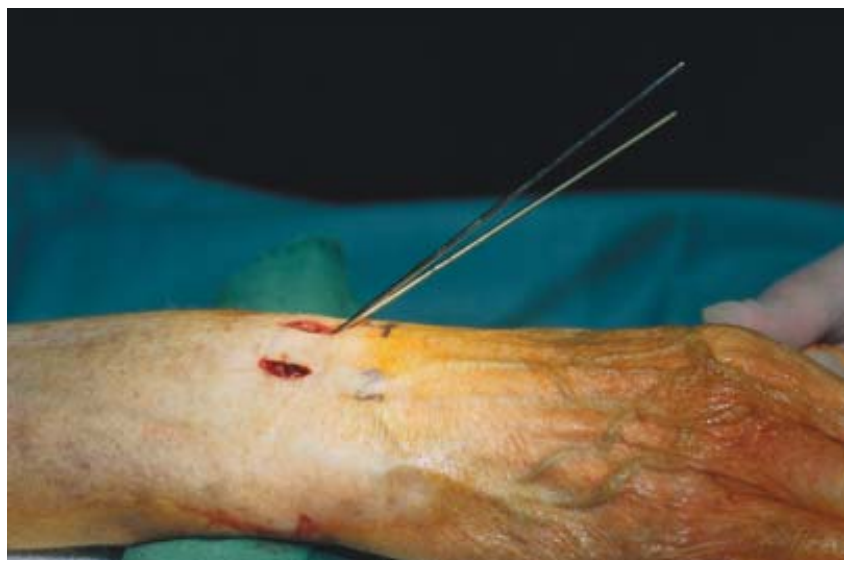

Abb.14 Versenkter erster Kirschner-Draht unter der Haut.
Zur Vermeidung von Sehnenrupturen oder Hautperforationen im Verlauf muss dem anschließenden Versenken der Kirschner-Drahtenden unter die Haut große Aufmerksamkeit geschenkt werden. Dabei wird mit einer spitzen Zange der nun festsitzende KirschnerDraht knapp über Sehnennniveau fixiert und mit einer zweiten Zange das Kirschner-Drahtende soweit nach dorsal umgebogen, dass dieses Ende nach einer weiteren halben Drehung des GewindeKirschner-Drahtes genau parallel zur Hautoberfläche zu liegen kommt (Abb.13). Nach Kürzen des KirschnerDrahtendes 5-7 mm oberhalb der Biegungsstelle, wird dieser um $180^{\circ}$ weiter eingedreht und liegt damit unter Hautniveau (Abb.14). Nach Kontrolle der Blut- stillung erfolgt der Verschluss der Hautinzisionen mit Einzelknopfnähten. Um eine sichere Fixation postoperativ zu erreichen, wird ein feuchter Verband mit offenen Gazekompressen angelegt, darüber folgt eine radial umfassende Gipsschiene oder eine Handgelenksmanschette mit Klettverschluss (Abb.15).

Durch sorgfältiges Versenken der Kirschnerdrahtenden können störende Komplikationen weitgehend vermieden werden

Tip: Bei Frakturen welche drohen, zusätzlich nach Reposition nach palmar zu dislozieren, kann ein durch den Prozessus styloideus radii oder ein leicht radio-palmar durch den Frakturspalt eingebrachter vierter Kirschner-Draht zusätzliche
Stabilität bringen. Die Notwendigkeit eines solchen vierten Kirschner-Drahtes kann meist erst intraoperativ beurteilt werden.

\section{Nachbehandlung}

Bei der Nachbehandlung ist zu beachten, dass die Handgelenksmanschette bei jungen Patienten für vier, bei älteren Patienten für sechs Wochen zu tragen ist. Sie kann täglich für ergotherapeutische Übungen der Langfinger sowie für Körperpflege wie Duschen etc., ausgezogen werden. Ansonsten richtet sich die Nachbehandlung nach den Vorschriften für konservativ behandelte Frakturen. Das Handgelenk sollte nicht beübt werden, um Kirschner-Drahtdislokationen/-aus-

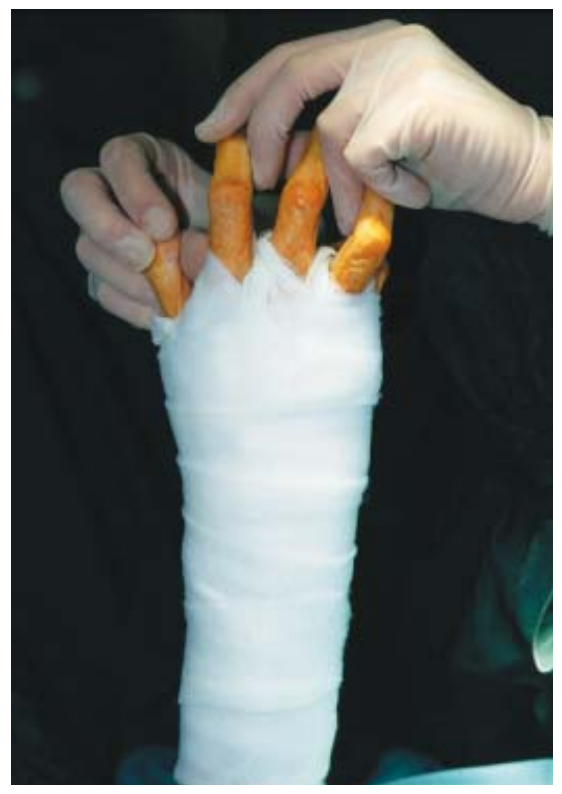

Abb.15 Feuchter Gazekompressen-Verband vor anlegen einer Gipsschiene oder Hangelenksmanschette.

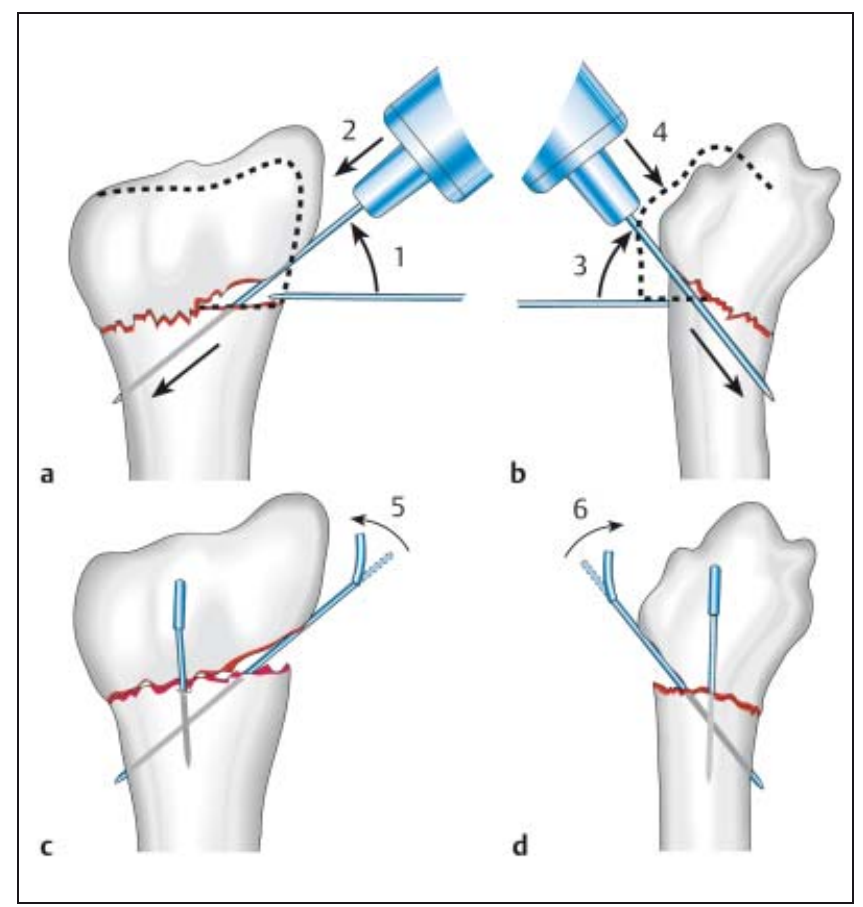

Abb.16 Schema der intrafokalen KDOsteosynthese. 
lockerungen sowie Sekundärdislokationen der Frakturfragmente zu vermeiden.

In instabilen Situationen, bei osteoporotischen Knochen und in allen Zweifelsfällen empfiehlt sich eine Röntgen-Verlaufskontrolle 8-10 Tage nach Operation zur frühzeitigen Erkennung einer Sekundärdislokation.

Nach sechs Wochen Nachbehandlung in der Handgelenksmanschette erfolgt eine klinische und radiologische Verlaufskontrolle. Bei sichtbarer Frakturkonsolidation kann die Kirschner-Draht-Entfernung in Lokalanästhesie geplant werden, welche frühestens acht Wochen nach Implantation erfolgen sollte.

\section{Osteosynthesematerialentfernung}

Dieser Eingriff kann problemlos ambulant in Lokalanästhesie erfolgen. Nach sterilem Abdecken werden die oft nur noch knapp sichtbaren ehemaligen Inzisionsstellen mit einem Lokalanästhetikum unterspritzt. Nach erneutem Durchführen der Hautinzisionen über eine Länge von 7-10 mm erfolgt analog der Implantation die tiefere Präparation stumpf mit zwei spitzen Moskitoklemmen bis auf die Kirschner-Drahtenden. Nach Fassen derselben mit einer spitzen Zange können diese anschließend problemlos ausgedreht werden. Nach Kontrolle der Blutstillung erfolgt der Hautverschluss und ein Absorptionsverband für 48 Stunden (Abb.17-19).

Ein grosser Vorteil der Kirschnerdrahtosteosynthese ist die Möglichkeit der Osteosynthesematerialentfernung in Lokalanästhesie.

\section{Fehlerquellen/Gefahren}

Trotz der relativ einfachen, aber wichtigen Operationstechnik birgt die Methode Fehlerquellen, Komplikationen und Gefahren (Tab.3). Durch eine ungenügende Präparation der Weichteile können Sehnen penetriert und pexiert werden, was zu erheblichen Beschwerden bis hin zu Sehnenrupturen im Verlauf führen kann.

Werden die Kirschner-Drähte nicht durch den Frakturspalt eingebracht, können sich diese nicht an zwei Kortikales abstützen, was zu einer erhöhten Rate an Sekundärdislokationen führen kann. Ein weiterer Grund für Sekundärdislokationen kann in der Verwendung von zu dünnen Drähten oder zu kurzem Gewinde liegen. Da die Kirschner-Draht-Osteosyn-

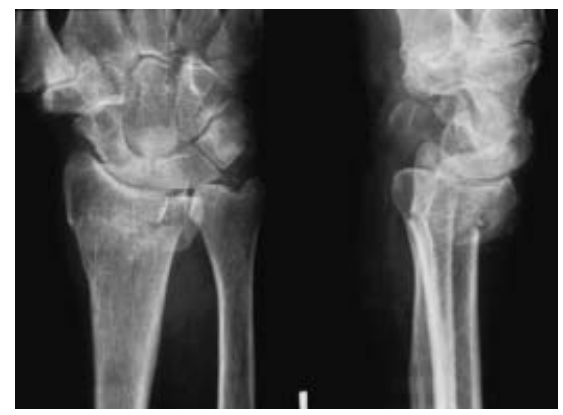

Abb.17 71-jährige Patientin, Sturz auf Eis. Unfallbild.

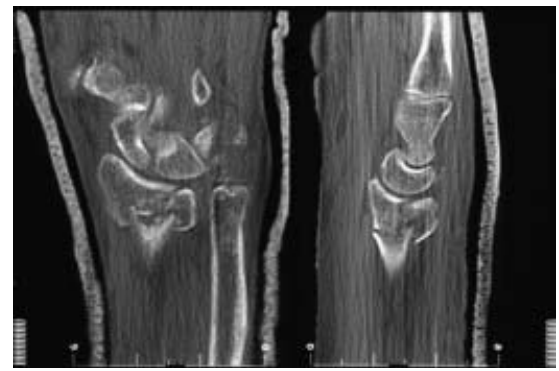

Abb.18 Präoperative Computertomographie.

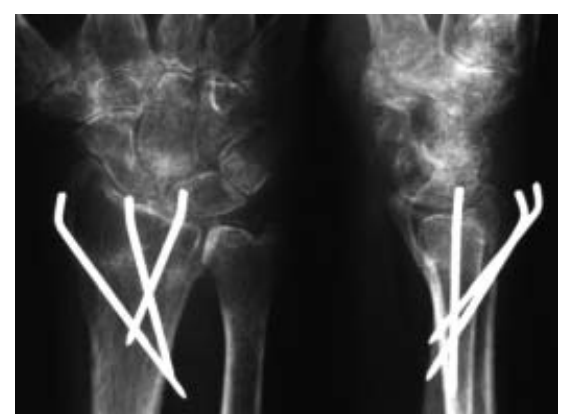

Abb.19 Kontrollbild nach 6 Wochen - vor KD-Entfernung in Lokalanästhesie.

these nicht als bewegungsstabil bezeichnet werden kann, muss eine zusätzliche äußere Fixation mit Gips oder Handgelenksmanschette erfolgen um Sekundärdislokationen auf ein Minimum zu beschränken.

Werden die Drähte zu flach eingeführt, das heißt in einem Winkel von weniger als $45^{\circ}$ zur Radiusschaftachse, drohen diese an der Gegenkortikalis abzugleiten und im Markraum zu wenig Halt zu finden.

Weichteilprobleme können ihre Ursache in einem zu kurzen oder zu langen Abschneiden der Kirschner-Drahtenden haben, ebenso wie ungenügendes Umbiegen derselben und ungenügendes Versenken unter der Haut. Perforationen der Haut können ihre Ursache zudem im zu engen Anlegen der äußeren Fixa-
Tab. 3 Fehlerquellen

— ungenügende Weichteilpräparation

- Einbringen der Drähte nicht in den Frakturspalt

- Verwendung von zu dünnen Drähten

- Verwendung von zu kurzem Gewinde

— zu flaches Einführen der Drähte in den Markraum

— ungenügendes Kürzen, Umbiegen und Versenken der KD-Enden unter der Haut - Ungenügende Fixation von außen - Druck von außen durch Manschette zu frühes Entfernen der Drähte

tion haben, sei es durch einen Gips oder eine Handgelenksmanschette. Ein direkter Druck auf die Kirschner-Drahtenden von außen muss vermieden werden. Im Falle von Kirschner-Drahtperforationen durch die Haut sollte eine regelmäßige Wundkontrolle erfolgen. Die Kirschner-Drähte können unter Umständen problemlos belassen werden, eine sich in die Tiefe entwickelnde Infektion muss aber unbedingt vermieden werden. Bei korrekter Nachbehandlung sind Pseudarthrosebildungen oder Algodystrophien selten.

Im Falle von Hautperforationen durch die Kirschnerdrähte können diese meist problemlos belassen werden, eine sorgfältige Wundpflege ist aber unerlässlich.

\section{Schlussfolgerung}

Für die Versorgung von distalen Radiusfrakturen konkurrieren sich verschiedene Methoden. Als Bindeglied zwischen der konservativen Frakturbehandlung und den Osteosynthese-Verfahren mit Platten oder Fixateur externe hat die KirschnerDraht-Osteosynthese ihren festen Platz. Bei korrekter Indikationsstellung für Monoblock-Frakturen ohne wesentliche Gelenksbeteiligung, korrekter Operationstechnik und adäquater Nachbehandlung besticht die Methode durch ihre Einfachheit. Sie ist schnell, kostengünstig und vermag viele Vorteile zu bringen.

\section{Literatur}

${ }^{1}$ Kapandji A. L'osteosynthèse par double embrochade intra-focal. Ann Chir 1976 ; 30 (1112) : 903-908

${ }^{2}$ Walton N P, Brammar T J, Hutchinson J, Raj D, Coleman N P. Treatment of unstable distal radial fractures by intrafocal, intramedullary K-wires. Injury 2001; 32 (5): 383-389

${ }^{3}$ Mittelmeier W, Braun C, Schafer R. The Kapandji technique for fixation of distal radius fractures - a biomechanical comparison of primary stability. Arch Orthop Trauma Surg 2001; 121 (3): 135-138 
${ }^{4}$ Jeyam M, Andrew J G, Muir L T S W, Mcgovern $A$. Controlled trial of distal radial fractures treated with a resobable bone minera substitute. J Hand Surg Br 2002; 27 (2): 146149

${ }^{5}$ Fritz T, Wersching D, Klavora R, Krieglstein C, Friedl W. Combined Kirschner wire fixation in the treatment of Colles fractures. A prospective, controlled trial. Arch Orthop Trauma Surg 1999; 119 (3-4): 171-178

${ }^{6}$ Board T, Kocialkowski A, Andrew G. Does Kapandji wiring help in older patients? A retrospective comparative review of displaced intra-articular distal radial fracutres in patients over 55 years. Injury 1999; 30 (10): 663-669

${ }^{7}$ Habernek H, Schmid L. Technique and results of modfied percutaneous bore wire osteosynthesis of the distal radius. Unfallchirurg 199295 (7): 339-343

${ }^{8}$ Kapandji A. Reduction effect ARUM-type intra-focal pins in the osteosynthesis of fractures of the lower end of the radius. Ann Chir Main Memb Super. 1991; 10 (2): 138-145

${ }^{9}$ Oestern H J, Huls E. Differential treatment of fractures of the distal radius. Zentralbl Chir 1994; 119 (8): 521-532

${ }^{10}$ Hoffmann T F, Ruppert R, Renneker D. Treatment results after surgical therapy of distal radius fractures. Unfallchirurg 1994; 97 (9): 472-477

${ }^{11}$ Hoel G, Kapandji A I. Osteosynthesis using intra-focal pins of anteriorly dislocated fractures of the inferior radial epiphysis. Ann Chi Main Memb Super 1995; 14 (3): 142-156
Dr. med. Thomas Klaus Hotz Leitender Arzt Traumatologie

Departement Chirurgie Kantonsspital

Brauerstraße 15

CH-8401 Winterthur 